\title{
Krebszellen mögen Zucker, aber noch mehr lieben sie Fett und tierisches Eiweiß
}

\author{
Ludwig Manfred Jacob ${ }^{1}$ \\ Nicole Weis ${ }^{2}$
}

\section{Zusammenfassung}

Zweifelsohne hat unsere Ernährung Einfluss auf das Krebsgeschehen. Allerdings gehen die Meinungen über die richtige Antikrebsdiät oft weit auseinander. In den letzten Jahren wurde eine proteinreiche Abwandlung der ursprünglichen ketogenen Diät als angeblich wirksame und wissenschaftlich belegte Krebsdiät propagiert. Bei genauer Sicht der epidemiologischen und ernährungswissenschaftlichen Studienlage scheint jedoch höchste Vorsicht geboten. Die These, eine „ketogene Diät“ könne den Tumor „aushungern“ und das Überleben verlängern, ist wissenschaftlich nicht belegt. Im Labor werden Krebszellen bekanntlich nicht in Zuckerwasser, sondern in Kälber- oder Rinderserum gezüchtet. Der hohe Anteil an tierischem Eiweiß in der neuen ketogenen Diät dient Krebszellen als Brenn- und Baustoff, belastet den Stoffwechsel und wirkt insulinogen. Das viele Fett nährt Adipozyten wie Krebszellen gleichermaßen und kann auf Dauer eine Insulinresistenz fördern. Krebszellen versorgen sich durch die Überexpression von Glukosetransportern noch mit Glukose, wenn der Mensch schon lange an Hypoglykämie verstorben ist. Die starke Kohlenhydratrestriktion senkt also nur die Lebensqualität und erhöht das Risiko psychischer und metabolischer Störungen, wie sie für extreme LowCarb-Diäten bekannt sind. Neue Erkenntnisse zeigen, dass Krebszellen einen gesteigerten Fettstoffwechsel und eine erhöhte $\beta$-Oxidation aufweisen und Fettsäuren zur mitochondrialen Entkopplung und dem Warburg-Effekt beitragen. Die als Antikrebsdiät propagierte Ernährungsweise steht im Widerspruch zu ernährungswissenschaftlichen und medizinischen Goldstandards.

Schlüsselwörter: Krebs, ketogene Diät, glykämischer Index, Insulin-Index, WarburgEffekt

\section{Einleitung}

Die ketogene Diät wurde ursprünglich zu Beginn der 1920er-Jahre zur Therapie medikamentös schwer einstellbarer Epilepsie in der Pädiatrie entwickelt und ist per definitionem eine fettreiche, proteinnormale, sehr kohlenhydratarme, isokalorische Ernährungsform, die nur unter erfahrener Aufsicht erfolgen sollte [1]. Bei dieser Diät wird bewusst eine Ketose, bei der die Energiegewinnung über Ketonkörper erfolgt, angestrebt. Dies gelingt nur unter strenger Kohlenhydratrestriktion und mo-

\footnotetext{
1 Dr. Jacob's Institut, Heidesheim a. Rh.

2 Redaktion Deutsche Zeitschrift für Onkologie, Leonberg
}

derater Proteinzufuhr, denn die Aminosäuren sind zum Großteil glukogen und können zur Glukoneogenese genutzt werden.

Eine Ketose entsteht durch eine stark gesteigerte Fettverbrennung, die zur Bildung der drei Ketonkörper Acetoacetat, 3beta-Hydroxybutyrat und Aceton führt. Die Ketose ist das Ergebnis eines Mangels an verfügbarer Glukose in den Zellen, wie z.B. beim Diabetiker, bei langen Fastenperioden oder beim Verhungern. Es ist damit fraglich, ob dies für den Menschen ein wünschenswerter, gesunder und natürlicher Zustand ist.

Ketonkörper stimulieren die Insulinfreisetzung [16, 26, 27], fördern eine metabolische Azidose, erzeugen Sauer- stoffradikale und sind eine Ursache von Lipidperoxidation [17-19]. Diabetiker weisen meistens eine gesteigerte Ketonkörpersynthese auf. Lipidperoxidation und die Erzeugung von Sauerstoffradikalen spielen eine wichtige Rolle bei Gefäßerkrankungen bei Diabetikern [18].

Die echte ketogene Diät wird aus verständlichen Gründen seit der Erfindung wirkungsvoller Pharmaka sehr selten angewendet. Die neue Form der ketogenen Diät in der alternativen Krebstherapie enthält nun zusätzlich eine besonders hohe Menge von 1,4 g Protein/kg KG, zumeist tierischen Ursprungs. Bei dem hohen Proteinanteil wird eine echte Ketose häufig nicht erreicht. Daher verdient diese neue ketogene Diät korrekterweise die Bezeichnung „Fett-Tierprotein-Kost“. Denn der Proteinanteil liegt weit über dem der ursprünglichen echten ketogenen Diät zur Epilepsie-Therapie und auch weit über dem menschlichen Bedarf sowie den seriösen Empfehlungen der Ernährungswissenschaft.

Es ist kein Geheimnis, dass Krebszellen im Labor nicht in Zuckerwasser, sondern zumeist in Rinder- oder Kälberserum wachsen $[29,50]$. Auch die Aminosäure Glutamin wird zu Wachstumszwecken gerne Krebszellkulturen zugefügt.

Protein wird nicht nur in der Leber, sondern auch in Krebszellen zu Glukose verarbeitet, wobei die Last an potenziell toxischem Stickstoff (Harnstoffzyklus, Ammoniak) und fixen Säuren erheblich steigt und auf Dauer für vielseitige Stoffwechselprobleme sorgt. Gleichzeitig ist Protein natürlich auch ein hervorragender Bau- 
stoff (Proteinsynthese) für die Tumorproliferation. Bei Glukosemangel können Krebszellen auch Glutamin zu Ammoniak und Milchsäure (Glutaminolyse) abbauen, um die Aminosäure als Kohlenstoffquelle zu nutzen. Bereits 1915 berichtete Janney, dass 3,5 g Glukose aus 6,25 g verzehrtem Fleischprotein hergestellt werden können [20]. $100 \mathrm{~g}$ Rinderprotein liefert mit einer gewissen Verzögerung also $56 \mathrm{~g}$ Glukose. Für andere Proteine liegt die Glukoseausbeute zwischen $50-84 \mathrm{~g}$. Darüber hinaus entsteht eine erhebliche Stoffwechselbelastung mit Schwefelsäure, Stickstoff und Ammoniak. Vor allem tierisches Protein enthält viel Methionin, was beim Abbau zu oxidativem Stress und zur Bildung von Homocystein führt.

Der Grund für fett- und proteinreiche Antikrebsdiäten ist die unbewiesene These, durch eine starke Kohlenhydratreduktion die Verfügbarkeit von Glukose im Blut zu senken und Tumorzellen quasi „auszuhungern“. Eine Vielzahl von Daten belegt allerdings, dass Tumorzellen Glukosetransporter überexprimieren, die ihre halbmaximale Aktivität bereits im niedrigen Glukosebereich um und unter $2 \mathrm{mmol}$ (18 mg/dl) erreichen (GLUT1, GLUT3). Es ist deshalb davon auszugehen, dass eine Glukoseabsenkung unter physiologischen Bedingungen nicht ausreicht, um die Glukoseversorgung maligner Zellen zu gefährden $[1,28,30,32,37]$.

\section{Steak setzt deutlich mehr anaboles Insulin frei als Pasta al dente}

Das metabolische Syndrom und die nichtalkoholische Fettlebererkrankung bilden die Frühsymptomatik unserer Zivilisationserkrankungen, in deren Kern Überernährung, Bewegungsmangel, Insulinresistenz und Hyperinsulinämie pathogenetisch wirken. Das viszerale und intrahepatische Fett muss dabei nicht allzu viel sein, um eine Insulinresistenz und Hyperinsulinämie zu bewirken, und sogar Schlanke können dank der modernen Lebensweise immer häufiger eine nicht alkoholbedingte Fettleber (NAFLD) haben. Mit einer Prävalenz von fast $50 \%$ in den USA [50] wird hierzulande die metabo- lische Bedeutung der NAFLD, die immer mit einer Insulinresistenz einhergeht, noch massiv unterschätzt.

Die Insulinresistenz führt dazu, dass zwar die Körperzellen gegenüber der blutzuckerregulierenden Insulinwirkung resistent werden, jedoch die kompensatorische Hyperinsulinämie die anderen Insulinwirkungen noch verstärkt: Insulin wirkt stark anabol und fördert damit nicht nur Fettstoffwechselstörungen und Adipositas, sondern auch die Krebsentstehung und Tumorproliferation. Insulin erhöht auch die Cholesterinsynthese (Bildung der Sexualhormone) sowie den Sympathikotonus (Puls- und Blutdruckerhöhung).

Auch die krebsfördernde Insulinwirkung wird als Argument für die „ketogene Diät“ angeführt, ohne grundlegende wissenschaftliche Tatsachen wie den FoodInsulin-Index FII zur Kenntnis zu nehmen. In der bisher umfangreichsten Untersuchung [3] des Effekts von Lebensmitteln auf die Insulinsekretion (Insulinindex) ergab sich, dass der Kohlenhydratgehalt und der glykämische Index (GI) nur die Insulinsekretion für kohlenhydratreiche Nahrungsmittel vorhersagen konnten. Selbst die glykämische Last erklärte weniger als die Hälfte der erreichten Insulinblutwerte, war aber noch der wertvollste Prädiktor. Der Insulinindex ist das Ergebnis der Arbeitsgruppe um Jennie Brand-Miller, eine der führenden GI-Forscherinnen der Welt.

Besonders insulinogen wirkt laut Insulinindex die Kombination von schnell verfügbaren Kohlenhydraten wie Zucker/ Weißmehl mit Tierprotein: z.B. Gummi-

Dass $1000 \mathrm{~kJ}$ (333 g) fettarmer Fisch (FII 43) und 1000 kJ (158 g) Steak (FII 37) zu einer wesentlich höheren Insulinausschüttung als $1000 \mathrm{~kJ}$ (200 g) Pasta al dente (FII 29) führen, zeigt die Gefahr dieser pseudowissenschaftlichen Ernährungsratschläge, die Pasta verbieten und Schweinebraten empfehlen. Wer übrigens nun $227 \mathrm{~g}$ Tofu isst, nimmt zwar auch $1000 \mathrm{KJ}$ und $27 \mathrm{~g}$ Protein auf, hat aber eine wesentlich niedrigere Insulinausschüttung (FII 21). Die Auswahl der Lebensmittel bedarf also einer differenzierteren Betrachtung. bärchen, Milchschokolade, Früchtejoghurt, Pizza, Schnitzel mit Pommes, Steak mit Kartoffeln, Cornflakes in Milch, Müsli mit Zucker oder Rosinen in Milch, Cappuccino, Milchshakes, Wurst- und Käsebrötchen, Hamburger und anderes Fast Food. Aufgrund der hohen Suchtpotenz der Insulinwirkung wundert es nicht, dass es sich hierbei um die beliebtesten Mahlzeiten handelt.

Fälschlicherweise werden Kohlenhydrate für die Insulinresistenz, Hyperinsulinämie und deren Endstadium Diabetes mellitus Typ 2 verantwortlich gemacht. Diese spielen jedoch im Vergleich mit tierischem Protein und Fett eine stark untergeordnete Rolle. In der EPIC-Studie mit 38.094 niederländischen Teilnehmern [44] wurden in einem Follow-up von 10 Jahren die Risiken einer Diabeteserkrankung bestimmt: Tierprotein führte zu einem $118 \%$ höheren Risiko (höchstes versus niedrigstes Quartil), Pflanzenprotein zeigte keine Korrelation. Dagegen führte eine hohe glykämische Last nur zu einer Risikoerhöhung von $27 \%$, hohe GI-Werte in der Ernährung um $8 \%$. Ballaststoffe senkten sogar das Risiko um $8 \%$, besonders viele Kohlenhydrate erhöhten es nur um $15 \%$, Stärke um $25 \%$.

In einer anderen großen Metaanalyse der Harvard School of Public Health mit insgesamt 442.101 Teilnehmern erhöhte rotes Fleisch das Diabetesrisiko am stärksten [33]. Selbst nachdem bekannte Risikofaktoren (Alter, BMI und andere Lebensstil- und Ernährungsfaktoren) statistisch berücksichtigt worden waren, führten täglich $100 \mathrm{~g}$ rotes, unverarbeitetes Fleisch zu einem 19\% höheren Risiko. Bereits täglich $50 \mathrm{~g}$ verarbeitetes Fleisch erhöhte das Diabetesrisiko um $51 \%$. Wer dagegen Fleisch mit Nüssen ersetzte, senkte sein Diabetesrisiko um $21 \%$; wer es mit Vollkornprodukten ersetzte, sogar um $23 \%$. Einem Krebspatienten zu „unbegrenzt“ Fleisch und Wurstwaren zu raten, fördert nicht nur Krebs, sondern auch die Entwicklung einer Insulinresistenz und Hyperinsulinämie [33].

Einfache und schnell verfügbare Kohlenhydrate (z.B. Zucker, Weißmehl) gelten im Gegensatz zu komplexen Kohlenhydraten mit Recht als ungesund, weil sie zu 
einem schnellen Blutzuckeranstieg und einer hohen Insulinantwort, besonders in Kombination von Proteinen, führen. Durch eine ballaststoffreiche Kost mit langsam verwertbaren Kohlenhydraten können jedoch Blutzucker- und Insulinspitzen vermieden werden, ohne den Tumor mit zu viel Eiweißbausteinen, anabolem Insulin sowie IGF-Wachstumsfaktoren zu versorgen und die Leber durch ein Übermaß an Eiweiß mit Ammoniak und anderen Endotoxinen einer fleischreichen Kost zu belasten. Der Leberstoffwechsel von Krebskranken ist ohnehin durch den Abbau der Milchsäure und anderer toxischer Stoffwechselprodukte des Tumors überfordert.

\section{Krebszellen mögen Zucker, aber lieben Fett}

Ein Stoffwechsel mit Insulinresistenz, Hyperinsulinämie und mitochondrialer Zytopathie - also der typische „Zivilisationsstoffwechsel“ - hat alle Voraussetzungen für eine verstärkte aerobe Glykolyse. Dies ist für Krebszellen typisch, wird im „Warburg-Effekt" beschrieben und häufig als Rechtfertigung für eine „ketogene Diät“ angeführt.

Otto Warburg vermutete 1956, dass Krebszellen dadurch entstehen, dass die oxidative Phosphorylierung in der Atmungskette permanent gehemmt und geschädigt wird und die anaerobe Fermentation nicht mehr durch Sauerstoff gehemmt wird (Pasteur-Effekt). Er prägte den Begriff der „aeroben Glykolyse“, d.h. der Vergärung von Glukose zu Milchsäure trotz Anwesenheit von Sauerstoff. Tatsächlich ist für Krebszellen häufig ein vermehrtes Aufkommen von Milchsäure charakteristisch.

Bei Krebszellen ist die Zellatmung stark eingeschränkt und wird durch eine aerobe Glykolyse (Warburg-Effekt) zum Teil ersetzt. Hierbei korreliert das Ausmaß der Milchsäureproduktion positiv mit der Malignität und Radio/Chemotherapie-Resistenz des Tumors sowie der schlechten Prognose [48, 49]. Eine Ausleitung der Milchsäure, die den Tumor vor Immunabwehr, Radio- und Chemotherapie schützt und die Invasion fördert, könnte daher ein bisher übersehener zentraler Bestandteil der Krebstherapie sein. Zudem setzt der Körper in einem sauren Milieu vermehrt entzündungsfördernde Substanzen wie NF-kappaB, TNF-alpha und COX-2 frei, welche den Knochenabbau beschleunigen und ein insgesamt proentzündliches, tumorfreundliches Milieu schaffen.

Die aerobe Glykolyse verschafft Krebszellen enorme Selektionsvorteile, indem sie ihre Wachstumsnische stark übersäuern und so ihre Metastasierung vorantreiben. In einem Tumormodell wurde das Maß der Übersäuerung als der entscheidende Schritt vom lokalisierten Tumor zur aggressiven Invasion ermittelt [34].

Andere Enzyme und Stoffwechselvorgänge scheinen eine höhere Bedeutung zu haben. Zahlreiche Studien finden sich zum Glyoxalase-Komplex. Durch den Glyoxalase-Enzym-Komplex produzieren aggressive Krebsstammzellen große Mengen schwer abbaubarer linksdrehender Milchsäure. Glyoxalase-1 wird u.a. durch Curcumin [39] gehemmt und gilt als neues mögliches Therapieziel bei der Leukämietherapie [45]. Durch die Hemmung kann Methylglyoxal nicht zu linksdrehender Milchsäure abgebaut werden und hat eine tumorstatische Wirkung.

Zahlreiche Studien weisen darüber hinaus auf die Bedeutung der LDH5 als einen interessanten Marker für die Milchsäureproduktion fortgeschrittener Tumore hin (z.B. [6, 23]). Eine wichtige Rolle bei der aeroben Glykolyse und dem damit verbundenen Krebswachstum spielt das Gen TKTL1 (transketolase-like-1). Die Aktivierung von TKTL1 in Krebszellen führt zu einer erhöhten Glukoseaufnahme, einer verstärkten Milchsäureproduktion sowie einem maligneren Phänotyp der Krebszellen. Zahlreiche Studien zeigen, dass eine erhöhte Expression von TKTL1 mit einer geringeren Überlebensrate, einer erhöhten Anzahl an Metastasen und Rezidiven und einer Resistenz gegenüber Chemo- und Strahlentherapien verbunden ist [6, 23]. Die klinische Bedeutung der TKTL1 wird nach wie vor kontrovers diskutiert. Sie gehört wohl wie die Glyoxalase und Fettsäuresynthase zu den wichtigen Enzymen des Tumorstoffwechsels, die aber nur in der Gesamtschau gedeutet werden können.
Daraus eine einseitige Ernährungsweise mit sehr hohem Fett- und Proteinanteil abzuleiten, kann durch die Studienlage nicht gerechtfertigt werden.

Denn Krebszellen können sich nicht nur durch die Vergärung von Zucker, sondern auch durch die wesentlich effizientere Oxidation von Fettsäuren ernähren. Eine fettreiche Ernährung versorgt auch Zellen mit mitochondrialer Dysfunktion und gestörter Atmungskette mit Energie und ist für Krebszellen eine optimale Energiequelle. Zusammenhänge siehe Abb. 1 .

Ein erniedrigtes mitochondriales Membranpotenzial und die mitochondriale Entkopplung der Atmungskette lösen in Leukämiezellen den Warburg-Effekt aus, induzieren eine Chemotherapie-Resistenz und verursachen im Zellstoffwechsel eine verstärkte aerobe Glykolyse und erhöhte Fettsäureoxidation [38]. Die mitochondriale Entkopplung wurde zu einem großen Teil über Milieufaktoren (Microenvironment) durch das entkoppelnde Protein 2 (UCP2) bewirkt. UCP2 fördert die Fettsäureoxidation, während die mitochondriale Oxidation von Pyruvat gehemmt wird [35]. Durch diesen Effekt sammelt sich in der Krebszelle verstärkt Milchsäure an, da der Abbauweg über Pyruvat gestört ist. Die intrazelluläre Ansammlung der Milchsäure ist auch für die

Entgegen der biologischen und biochemischen Vernunft sowie der epidemiologischen Datenlage soll man Krebs angeblich durch eine sehr fettund proteinreiche, kohlenhydratarme Ernährung bekämpfen können. Der Warburg-Effekt wird in diesem Zusammenhang zur Rechtfertigung herangezogen und missbraucht. Denn die Realität zeigt:

1. Eine fettreiche Ernährung fördert die mitochondriale Entkopplung und den Warburg-Effekt.

2. Krebszellen lieben Fett als energiereichsten Nährstoff und bevorzugen ihn zu ihrer Energiegewinnung. 3. Die „Fettsucht“ der Krebszellen geht so weit, dass Tumore sogar selbst Fettsäuren aufbauen und in vielen Tumoren die Fettsäure-Synthase (FAS) heraufreguliert ist. 


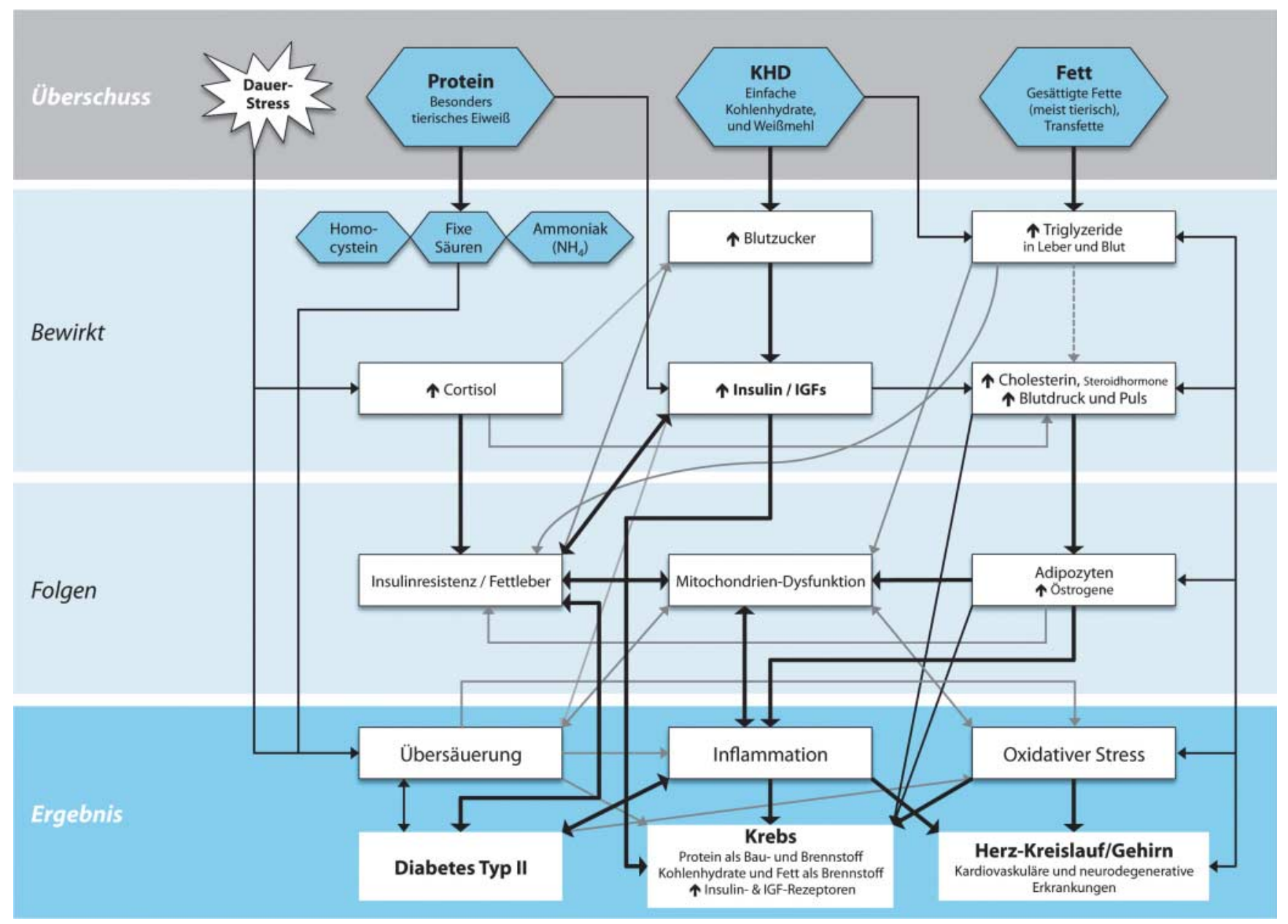

Abb. 1: Wirkung der Makronährstoffe.

Krebszelle schädlich, weshalb sie die Fettsäureoxidation vorzieht.

Auch andere Studien zeigen: Fettsäuren können eine mitochondriale Entkopplung verursachen und umgekehrt kann eine mitochondriale Entkopplung auch die Fettsäurenoxidation verstärken $[8,9$, 46].

Harper und Mitarbeiter vermuteten 2002 einen Zusammenhang zwischen gesteigertem Fettsäuren-Metabolismus und reduziertem mitochondrialen Membranpotenzial und postulierten, dass die Abnahme des elektrochemischen Gradienten in den Mitochondrien einen metabolischen Wechsel zu energiedichterem Substrat, d.h. Fettsäuren, darstellt [14].

Mit anderen Worten: Der Warburg-Effekt tritt auf, weil die Mitochondrien in Krebszellen die Fettsäurenoxidation bevorzugen und dies zur mitochondrialen Entkopplung führt [38].
Fettsäuren sind essenziell für die Energiegewinnung in Krebszellen. So können Leukämiezellen durch eine pharmakologische Hemmung der Fettsäurenoxidation in der Proliferation gehemmt und in ihnen eine Apoptose induziert werden. Auch eine Hemmung der FAS machte die Leukämiezellen Apoptose-anfällig, was zeigt, dass sich Krebszellen mit Fettsäuren zu schützen vermögen und diese daher selbst durch eine gesteigerte FAS-Aktivität synthetisieren [38].

Die Heraufregulation des FAS-Gens tritt in vielen verschiedenen Krebsarten auf. Die Überexpression des FAS-Gens reicht in Brustepithelzellen sogar aus, um die Zellen zu Krebszellen werden zu lassen. Die Überproduktion von Fettsäuren begünstigt offenbar stark die Progression und das Überleben von Krebszellen. FAS gilt bei Prostatakrebs als ein mögliches Onkogen [4], wird bei Brustkrebs herauf- reguliert [15] und gilt z.B. beim Ovarialkarzinom als ein Indikator für eine besonders schlechte Prognose [12].

Rotondo und Davidson [36] gehen weiter und zeigen: Durch eine Hemmung des Fettsäuretransports in die Mitochondrien mittels Carnitin-Palmitoyltransferase-1 (CPT-1) können Brustkrebszellen getötet werden. Lungenkrebszellen überexprimieren das CPT1-Gen 10- bis 100-fach. Auch die Östrogen-Rezeptor-positive Brustkrebszelllinie MCF-7 überexprimiert das CPT1-Gen und steigert so ihre Fettsäurenoxidation und ATP-Produktion.

Tumore haben eine gesteigerte Zuckerverwertung. Doch insbesondere langsam wachsende Tumore wie Prostatatumore haben eine stark gesteigerte Fettsäurenoxidation. Diese liefert sowohl ATP als auch Acetyl-CoA, welches Ausgangsstoff der Cholesterin- und Sexualhormon-Synthese ist. Cholesterin und Sexualhormone 
erhöhen das Risiko der hormonabhängigen Krebsarten (Mamma- und Prostatakarzinom). Die Fettsäureoxidation dominiert beim Prostatakrebs deutlich über die Glykolyse [25].

Im Mäusemodell konnten Khasawneh und Mitarbeiter [22] zeigen, das eine fettreiche Ernährung über eine Aktivierung von Onkogenen und proentzündlichen Prozessen die Tumorpromotion sowie die Expression von Genen förderte, welche die mitochondriale beta-Oxidation von Fettsäuren steigern.

Die Fettsäurenoxidation ist für die Krebszelle eine saubere Energiequelle im Gegensatz zur Glykolyse, welche die Zelle ansäuert und dazu zwingt, die Milchsäure energieaufwendig nach außen zu pumpen. Die wenigen aufgezeigten Studien sind eine kleine Auswahl, die zeigen, dass sich Krebszellen logischerweise nicht auf einen Weg der Energiegewinnung verlassen und sich in den allermeisten Fällen nicht mit einer kohlenhydratarmen, aber fettreichen Ernährung aushungern lassen. Vielmehr wirkt eine solche Ernährung krebsfördernd und stoffwechselschädigend.

\section{Brauchen wir mehr Fett und Protein?}

Die Deutschen halten sich für gesundheitsbewusst, haben aber objektiv die wenigsten gesunden Lebensjahre in Europa. Mit einem besonders hohen Konsum von Fett und Tierprotein sind sie die dicksten Europäer, wie die International Association for the Study of Obesity (IASO) feststellte: 75,4\% der Männer und 58,9\% der Frauen in Deutschland haben einen BMI über 25. In Italien dagegen gibt es die wenigsten Übergewichtigen und Adipösen.

Epidemiologisch ergibt sich folgendes Bild im Land der Pasta-LiebhaberInnen: In einer Studie mit 3367 postmenopausalen Italienerinnen stieg das Brustkrebsrisiko für die Gruppe mit dem höchsten Konsum an tierischem Eiweiß um den Faktor 3,78 und um den Faktor 3,47 für den höchsten Konsum an Fett im Vergleich zur Gruppe mit dem niedrigsten Verzehr. Kohlenhydrate gingen mit einem 58\% niedrigeren Risiko einher [43].

Deutsche nehmen im Durchschnitt $36 \%$ der Gesamtenergie in Form von Fetten überwiegend tierischen Ursprungs (Fleisch, Wurst, Eier, Fette, Milch, Käse) auf. Damit liegen sie ca. 10-12\% über den Empfehlungen der DGE. Die Fette in der deutschen Ernährung setzen sich hauptsächlich aus tierischen gesättigten und einfach ungesättigten sowie teilweise stark verarbeiteten Fetten (Transfettsäuren) zusammen.

Die empfohlene Proteinzufuhr liegt laut DGE bei $0,8 \mathrm{~g} / \mathrm{kg}$ KG und Tag, das entspricht $56 \mathrm{~g}$ bei einer $70 \mathrm{~kg}$ schweren Person. Die durchschnittliche Zufuhr in Deutschland liegt jedoch bei $74 \mathrm{~g} / \mathrm{Tag}$. Laut der Nationalen Verzehrsstudie II liegt die Proteinzufuhr von nur $11 \%$ der Männer und $15 \%$ der Frauen in Deutschland unter den Empfehlungen, im Durchschnitt sind die Deutschen mit 140\% insgesamt überversorgt. Die Hauptquelle ist Tierprotein (Fleisch, Wurst, Käse, Milch).

\section{Die angebliche ketogene Antikrebsdiät}

In einer aktuellen klinischen 3-monatigen Studie sollten 16 Studienteilnehmer mit fortgeschrittenem Krebsleiden einer solchen ketogenen Krebsdiät folgen. Nur fünf vollendeten die Studie, davon konnte nur ein Studienteilnehmer eine durchgängige Ketose erreichen [41]. Erstaunlicherweise fehlen in den Studienergebnissen selbst bei den Teilnehmern, welche die drei Monate absolvierten, zum Teil die zentralen Blutwerte für HBA1c und Trigylzeride zum Studienende. Auch die Schlussfolgerung der Studie ist bemerkenswert: „Diese Pilotstudie zeigt, dass eine ketogene Diät 
sogar für Patienten mit fortgeschrittener Krebserkrankung geeignet ist.“ Fakt ist, dass die Patienten vermehrt unter Verstopfung, Appetitlosigkeit, Übelkeit, Energiemangel und Schmerzen klagten, die Patienten weiter an Gewicht verloren und der Großteil des relativ jungen Patientenkollektivs (Durchschnitt 50,4 Jahren) die Studie aus diversen Gründen vorzeitig abbrach. Dies ist auch nicht verwunderlich, da bei kachektischen Patienten mit bereits katabolem Stoffwechsel eine hyperkalorische fett- und proteinreiche Ernährung ohne Kohlenhydratrestriktion angeraten ist.

Auch für nicht kachektische Krebspatienten mit früheren Krebsstadien ist diese Krebsdiät nicht geeignet: Sie erreichen bei der proteinreichen Form der ketogenen Diät meistens nicht einmal das zweifelhafte Therapieziel einer Ketose, viele erleiden jedoch eine Reduktion der Lebensqualität, Stoffwechselstörungen, Fressattacken, Depressionen und auf Dauer häufig eine unerwünschte Gewichtszunahme, die das Krebsgeschehen fördert. Bei prädisponierten Patienten ist der Weg von der Ketose zur lebensbedrohlichen Ketoazidose nicht sehr weit.

In dem Laienbuch „Krebszellen lieben Zucker - Patienten brauchen Fett“ [21], das von drei Biologen verfasst wurde, werden abschließend zahlreiche Empfehlungen ausgesprochen, die medizinischen und ernährungswissenschaftlich bestens etablierten Goldstandards widersprechen. Hier eine kleine Auswahl (Seite 228-235):

- Alle Getreidesorten sowie sehr viele

Gemüse- und Obstsorten sollten komplett gemieden werden.

- Zum „unbegrenzten“ Verzehr von rotem Fleisch und Wurst wird ausdrücklich geraten.

- „Faustregel: Je kg Körpergewicht essen Sie am Tag 2,5 g Fett, 1,4 g Eiweiß und 0,5 g Kohlenhydrate. Mindestens aber 175 g Fett.“ Das entspricht 1575 Kcal Fett.

Mit anderen Worten: Je dicker ein Mensch ist, desto fetter soll er essen. Wissenschaftlich gesichert ist: Je dicker man ist, desto wahrscheinlicher bekommt man Krebs oder ein Krebsrezidiv. Die hohe Zufuhr an
Fetten und Ölen erhöht stark die Kalorienzufuhr. Fette und Öle machen im Verhältnis zu ihrer hohen Kaloriendichte am wenigsten satt und sind eine der Hauptkalorienquellen. Überschüssige Fette werden auch nicht zwingend verbrannt, sondern von Fettzellen vorzugsweise eingelagert, was eher zu einer Zunahme der Leibesfülle als zu einer Abnahme des Tumors führt. Nur bei kachektischen Patienten kann eine solche Ernährungsweise sinnvoll sein - allerdings ohne Restriktion vollwertiger Kohlenhydrate.

Der World Cancer Research Fund (WCRF), der das globale Netzwerk von Anti-Krebs-Wohltätigkeitsorganisationen zur Vorbeugung und Therapie von Krebs koordiniert, und das American Institute for Cancer Research (AICR) kommen 2007 in ihrem zweiten Review (537 Seiten) zu Ernährung, Bewegung und Krebsvorbeugung zu folgenden offiziellen Empfehlungen:

- Seien Sie so schlank wie möglich innerhalb eines normalen Körpergewichts!

- Seien Sie täglich körperlich aktiv!

- Wählen Sie Essen mit niedriger Kaloriendichte! (D.h. wenig Fett und Softdrinks!)

- Essen Sie überwiegend Pflanzenkost, davon mindestens $600 \mathrm{~g}$ Gemüse und Obst sowie $25 \mathrm{~g}$ Ballaststoffe aus Vollkornprodukten und anderen Ballaststoffquellen. (Raffinierte Mehle und Zucker gelten nicht als Pflanzenkost.) Essen Sie möglichst wenig verarbeitetes Getreide und Leguminosen mit jeder Mahlzeit!

- Reduzieren Sie Fleisch auf maximal $300 \mathrm{~g}$ pro Woche und vermeiden Sie komplett verarbeitetes Fleisch (z.B. Wurstwaren).

Auf fünf Evidenzstufen wurden von dem weltweit maßgeblichen Gremium unter anderem folgende Risikoeinstufungen vorgenommen:

- Rotes Fleisch und verarbeitetes Fleisch fördert mit überzeugender Evidenz (höchste Evidenzstufe) Dickdarmkrebs, Ballaststoffe und ballaststoffhaltiges Getreide wirken wahrscheinlich pro- tektiv gegenüber Kolon- und Rektumkarzinom.

- Gemüse und Obst wirken wahrscheinlich protektiv gegenüber zahlreichen Krebsarten.

- Calciumreiche Ernährung und Milch erhöhen wahrscheinlich das Prostatakrebsrisiko.

- Kochsalz erhöht wahrscheinlich das Magenkrebsrisiko.

- Weder Getreide noch Zucker zeigen auf irgendeiner der fünf Evidenzstufen eine Risikoerhöhung von irgendeiner Krebserkrankung. (Dies soll nicht bedeuten, dass der übermäßige Verzehr isolierter Zucker von den Autoren für harmlos oder empfehlenswert gehalten wird.)

- Es erfolgt vermutlich eine Risikoerhöhung für Brust-, Lungen- und Dickdarmkrebs durch einen hohen Fettkonsum (suggestive evidence).

Neue Ergebnisse der europäischen EPICStudie belegen ebenfalls die hohe Risikokorrelation zwischen Brustkrebs und Fettkonsum. So verdoppelte ein hoher Fettkonsum (Gesamtfett) das Brustkrebsrisiko der deutschen EPIC-Teilnehmerinnen [42]. Bei Französinnen führten insbesondere Transfettsäuren aus industriell verarbeiteten Lebensmitteln zu einem 75\% höheren Brustkrebsrisiko [5]. Europaweit erhöhten gesättigte Fettsäuren nachweislich das Brustkrebsrisiko [13].

Der Umstand, dass viel Fett Menschen oft auch fett macht, ist bekannt und hängt insbesondere davon ab, wie viel sie sich bewegen. Wer sich viel bewegt, kann viel Fett verbrennen und wird nicht fett. Wer aber fettreich isst und sich nicht bewegt, wird irgendwann fett und erhöht damit stark sein Krebsrisiko.

Laut WCRF und AICR erhöht ein hoher Körperfettanteil mit überzeugender Evidenz das Risiko zahlreicher Krebsarten, während Bewegung das Risiko senkt. Nicht kachektischen, häufig übergewichtigen Krebskranken pauschal eine fettreiche „Antikrebs“-Ernährung zu verordnen, bei der die Fettzufuhr nach kg Körpergewicht berechnet wird, ist ein ärztlicher Kunstfehler.

Auch die jüngste große Metaanalyse zur manchmal behaupteten Verbindung 
von Kohlenhydraten, GI (= glykämischer Index) und GL (= glykämische Last) zum Kolonkarzinom kommt $\mathrm{zu}$ folgender Schlussfolgerung [2]: Diese Metaanalyse von Kohortenstudien unterstützt nicht eine eigenständige Risikoassoziation zwischen Ernährungsweisen mit viel Kohlenhydraten, hohem Glykämischen Index oder hoher Glykämischer Last und Kolonbzw. Rektumkarzinom. Anerkannt wird jedoch die Rolle von Adipositas, Bewegungsmangel, Typ-2-Diabetes und der damit verbundenen Insulinresistenz in der Ätiologie des Kolonkarzinoms.

Betrachten wir zum Abschluss noch die aktuellen S3-Leitlinien zum Prostatakrebs, die folgende Empfehlungen aussprechen:

- „Achten Sie auf eine gesunde Ernährung mit Schwerpunkt auf pflanzliche Produkte.

- Essen Sie jeden Tag verschiedene Obstund Gemüsesorten.

- Essen Sie lieber Vollkorn- als Weißmehlprodukte.

- Begrenzen Sie die Zufuhr von Fleischprodukten und rotem Fleisch.

- Streben Sie ein gesundes Gewicht an.

- Achten Sie auf eine gesunde Balance von Energie (Kalorien) und körperlicher Aktivität.

- Vermeiden oder reduzieren Sie Übergewicht und versuchen Sie, Ihr erreichtes gesundes Gewicht zu halten. Der gesündeste Weg, die Kalorienzufuhr zu reduzieren, ist eine Reduktion bzw. der Verzicht auf zusätzlichen Zucker, gesättigte oder Transfette und Alkohol. Alle diese Nahrungsmittel sind sehr energiereich und haben $\mathrm{zu}$ wenig oder keine essenziellen Nahrungsbestandteile wie Vitamine, Mineralien oder Ballaststoffe.“

Auch The American Heart Association der USA warnt u.a. offiziell: „Personen, die einer proteinreichen Diät folgen, riskieren einen Vitamin- und Mineralstoffmangel sowie Herz-, Nieren-, Knochen- und Leberleiden.“

Dean Ornish zeigte in der GEMINALStudie [31], welch großen Einfluss unser Lebenswandel auf Prostatakrebsgene hat.
Nach Prof. Leitzmann in „Ernährung bei Krebs“ [24] sollten bei Krebs 15\% der gesamten Energiezufuhr von $2000 \mathrm{kcal}$ aus Eiweiß (max. $75 \mathrm{~g}$ ), vorzugsweise nicht aus Fleisch, sondern aus laktovegetabiler Quelle und Fisch, ca. 25-30\% aus Fett (ca. 70-80 g, vorzugsweise Omega-3-Fettsäuren aus frischem Leinöl, Hanf, Raps, Fisch) und 55-60\% (275-300 g) aus langsam verwertbaren Kohlenhydraten bestehen. Noch sinnvoller ist wohl eine stoffwechsel- und stadienabhängige Ernährungsweise.

Die Patienten mit Prostatakrebs mussten ihren Lebensstil radikal umstellen: Sie ernährten sich mit einer fettarmen, pflanzlichen Vollwertkost, gingen 6 Tage pro Woche mindestens 30 Minuten spazieren, übten sich in Stressmanagement (wahlweise Yoga, Atemübungen, Meditation, Visualisierungsübungen, progressive Muskelentspannung) und nahmen einmal in der Woche an einer gemeinsamen Gruppensitzung teil. Die Mediziner entnahmen den Probanden sowohl vor als auch drei Monate nach dieser Intervention Biopsien der Prostata. In diesen konnten die Forscher Veränderungen der Expression für mehrere 100 Gene nachweisen. Für die Tumorbildung wichtige Gene wurden herunterreguliert, dagegen waren krebsbekämpfende Gene aktiver als vor der Lebensstilumstellung.

Dass diese Auswirkungen auch von prognostischer Relevanz sind, zeigte sich in einer Langzeitstudie. Denn es ließen sich die Ergebnisse von „active surveillance“ in einer weiteren klinischen Studie mit 93 Prostatakrebspatienten stark verbessern: Die Gruppe um Ornish zeigte, dass das Fortschreiten der Krankheit zu einer notwendigen invasiven Therapie durch die oben beschriebenen Maßnahmen um mindestens 2 Jahre verzögert wird [11]. Fünfmal mehr Teilnehmer der Kontrollgruppe mussten nach 2 Jahren eine invasive Therapie einsetzen als in der Ornish-Interventionsgruppe.

In einer kleineren Studie lebten $14 \mathrm{~Pa}-$ tienten mit rezidivierendem Prostatakrebs über 6 Monate von pflanzlicher Kost und praktizierten Stressmanagement. Bei 4 von 10 auswertbaren Patienten sank der PSA-Wert stark ab, 9 von 10 hatten eine drastische Verlängerung der PSA-Verdoppelungszeit [40].

Die sogenannte ketogene Diät steht damit im klaren Widerspruch zu internationalen Goldstandards der Ernährungsempfehlungen und Medizin. Sie kann sich daher auf keine seriöse breite wissenschaftliche Grundlage berufen, sondern muss möglicherweise als „Kunstfehler“ angesehen werden, sollte sie von Ärzten empfohlen werden.

Die These, ein Tumor ließe sich durch eine „ketogene Diät“ aushungern, ist naiv. Der Tumor reagiert mit der Überexpression von Glukosetransportern und kann sich noch Brenn- und Baustoffe aus dem Blut holen, wenn sein Wirt schon lange an Hypoglykämie verstorben ist. Über das viele Fett freuen sich Adipozyten wie Krebszellen gleichermaßen, über das Tierprotein die Krebszellen. Die starke Kohlenhydratrestriktion senkt die Lebensqualität und erhöht das akute Risiko psychischer und metabolischer Störungen, wie sie für extreme Low-Carb-Diäten bekannt sind.

\section{Fakten statt Mythen}

Bemerkenswerterweise müssen zur Rechtfertigung neuer Modediäten meistens Ernährungsweisen von Bevölkerungsgruppen herhalten, die entweder exotisch und wenig bekannt sind wie die Inuit oder ausgestorben sind wie die Steinzeitmenschen und damit wissenschaftlich nicht mehr objektiviert werden können. Wenig zielführend ist die Spekulation über unsere Steinzeit-Gene und daraus abgeleitete Diäten. Denn Steinzeitdiäten waren in ihrer Zusammensetzung sehr unterschiedlich und entsprachen im Wesentlichen den Möglichkeiten der jeweiligen Klimazone. Natürliche, nicht verarbeitete Nahrungsmittel und sehr viel Bewegung verbindet alle Naturvölker und ist Grundlage jeder gesunden Lebensweise.

Auf Seite 146 des Buches „Krebszellen lieben Zucker - Patienten brauchen Fett“ [21] heißt es, dass die Inuit (umgangssprachlich auch als Eskimos bezeichnet) mit ihrer Tierfett-Tierprotein-Kost traditionell selten an Krebs verstarben. Wenn 
man durchschnittlich nur 35 Jahre alt wird - wie die Grönland-Inuit - oder sogar das für einen Inuit hohe Durchschnittsalter von 48 Jahre wie die Alaska-Inuit (194951) erreicht, hat man kaum ausreichend Zeit, um an Krebs zu versterben, weil man vorher an Infektionskrankheiten wie TBC verstorben ist (vgl. Arctic Human Development Report, 2004, Arctic Demography, Bogoyavlenskiy und Siggner).

Die Inuit wanderten vor zehntausenden von Jahren aus Asien nach Grönland (Teil Dänemarks), Kanada und Alaska ein. Sie leben traditionell vom Fischen und Jagen, ernähren sich reichlich von „gesundem“ Fisch und befolgen aufgrund gegebener Ressourcen eine sehr fett- und eiweißreiche Diät. Inuit decken im Schnitt 75\% ihrer täglichen Kalorienzufuhr durch Fett. Im Jahr 2005 zeigte eine Inuit-Studie, dass die fischreiche Ernährung zwar zu hohen Omega-3-Fettsäure-Spiegeln im Blut führt, aber die Inuit nicht vor Herz-KreislaufErkrankungen schützt [7]. Vielmehr litten $26 \%$ der über 55-jährigen Inuit an HerzKreislauf-Erkrankungen. Die kanadischen Inuit haben auch heute noch mit ihrer Fett-Tierproteinreichen Kost die kürzeste Lebenserwartung (Männer 64 Jahre, Frauen 70) und die meisten Krebs- und Herz-Kreislauf-Erkrankungen aller Kanadier.

Bleiben wir weiter bei wissenschaftlich nachprüfbaren Fakten und betrachten die Lebenserwartung der nicht ausgewanderten Asiaten in den entwickelten Staaten Asiens mit überwiegend pflanzlicher und kohlenhydratreicher Kost und der weltweit höchsten Lebenserwartung: Japan 81 Jahre, Singapur 82 Jahre, etc. Die Menschen auf Okinawa haben die höchste Lebenserwartung in Japan und in der Welt (Männer 78 Jahre, Frauen 86 Jahre). Und sie sterben $\mathrm{zu}$ 80-90\% seltener an Herz-Kreislauf-Erkrankungen, Brust- und Prostatakrebs als Menschen in den USA oder Europa. Es ist dort noch üblich, mit weit über 100 Jahren an „Altersschwäche“ zu versterben. Sie ernähren sich traditionell überwiegend sehr pflanzen- und kohlenhydratreich sowie kalorien-, fettund proteinarm. (Die zunehmende Verwestlichung der Ernährung hat die Männer inzwischen bereits auf den letzten
Platz Japans zurückgeworfen.) Im Gegensatz zu den Ernährungsmythen, die im Buch „Krebszellen lieben Zucker - Patienten brauchen Fett“ [21] angeführt werden, sind die Okinawas Gegenstand des bislang längsten und am besten dokumentierten Projekts in der Forschung über 100-Jähriger (Kurzer Überblick: www.okicent.org).

Der ernährungswissenschaftliche Klassiker „Ernährung des Menschen“ [10] bestätigt, dass diese traditionellen Ernährungsweisen viel mehr der Natur des Menschen entsprechen als die moderne westliche Ernährung: „Die evolutionsgemäße Kost bedeutet heute für fast alle Menschen eine gemischte, jedoch stark überwiegend pflanzliche Kost, die somit als die natürliche Ernährung des Menschen angesehen werden kann." Dies zeigt sich nicht nur an der Entwicklungsgeschichte des Menschen, sondern noch heute u.a. an der Anatomie des menschlichen Gebisses und Darms sowie der Enzymausstattung. So hat der Mensch keine eigene Vitamin-CSynthese und verfügt über einen schlechten Harnsäure- und Cholesterinabbau. Der Darm eines pflanzenfressenden Gorillas und anderer Menschenaffen ähnelt bekanntlich sehr dem Darm eines Homo sapiens sapiens.

\section{Differenzierte Vorgehensweise}

Bei Krebskranken ist eine differenzierte Vorgehensweise erforderlich, welche das Tumorstadium, die persönliche Verträglichkeit und vor allem die individuelle Stoffwechselsituation berücksichtigt. Johanna Budwig hatte zu einer Zeit, in der Krebserkrankungen meistens in einem späten Stadium diagnostiziert wurden, mit einer Leinöl-Quark-Diät große Erfolge. Diese vielleicht einzig empfehlenswerte Form der Fett-Eiweiß-Kost ist für Patienten mit einer fortgeschrittenen Krebserkrankung, deren größter Feind die Kachexie ist, eine lebensverlängernde Ernährungsform. Allerdings sollte bei kachektischen Patienten keine Restriktion komplexer, „gesunder“ Kohlenhydrate vorgeschrieben, sondern zur Erhöhung der Kalorienzufuhr und des psychischen Wohlbefindens sogar angeraten werden.
Heute werden Krebserkrankungen häufig in einem relativ frühen Stadium entdeckt und behandelt. Einem Patienten mit Übergewicht und metabolischem Syndrom eine „Fett-Tierprotein-Kost“ zu verordnen, verstärkt seine Stoffwechselstörung und langfristig auch sein Übergewicht. Selbst ein hoher Anteil der angeblich besonders gesunden, aber sehr oxidationsempfindlichen Omega-3-Fettsäuren DHA und EPA im Blut erhöht zum Beispiel deutlich das Prostatakrebsrisiko, wie EPIC und Prostate Cancer Prevention Trial aufzeigen.

Weitere Infos zu den ernährungsphysiologischen Ursachen von Insulinresistenz und Hyperinsulinämie in der Pathogense von Krebs, Typ-2-Diabetes und Herz-Kreislauf-Erkrankungen sowie Ernährungsplan nach Dr. Jacob unter Berücksichtigung der Insulin-, Redox- und Säure-Basen-Balance: info@drjacobsinstitut.de.

Bei viszeraler Adipositas und metabolischem Syndrom, das immer mit einer Insulinresistenz und einer Hyperinsulinämie einhergeht, sind unbedingt ein Bewegungsprogramm sowie eine überwiegend pflanzliche, fettarme, ballaststoffreiche Ernährung anzuraten (Abb.2).

Autorenerklärung: LMJ erklärt, dass er Gesellschafter der Dr. Jacob's Medical $\mathrm{GmbH}$ ist. Beide Autoren erklären, dass keine finanziellen Interessenkonflikte im Zusammenhang mit diesem Beitrag bestehen.

\section{online:}

http://dx.doi.org/10.1055/s-0032-1314699

\section{Korrespondenzadresse}

Dr. med. Ludwig Manfred Jacob

Dr. Jacob's Institut

Egstedterstr. 46

55262 Heidesheim am Rhein

E-Mail: info@drjacobsinstitut.de

www.drjacobsinstitut.de 


\begin{tabular}{|c|c|c|c|}
\hline Dr. Jacobs Ernährungspyramide & & Lebensmittel & Empfehlungen \\
\hline & $\frac{\mathrm{g}}{\frac{\mathrm{g}}{\mathrm{d}}}$ & $\begin{array}{l}\text { Fleisch, Wurst, Chips, } \\
\text { Süßigkeiten, etc. }\end{array}$ & $\begin{array}{l}\text { Besser gar nicht, da .... } \\
\Theta \text { Wenig Vitalstoffe, aber viel Kalorien, Stoffwechselsäuren, } \\
\text { Oxidantien, Kanzerogene und andere Schadstoffe } \\
\Theta \text { Gesättigte und Transfette, Tierprotein, Kombination } \\
\text { von Tierprotein mit Zuckern } \\
\Rightarrow \text { Maximal 1-2 kleine Portionen pro Woche }\end{array}$ \\
\hline & 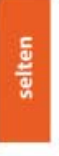 & $\begin{array}{l}\text { Milchprodukte, Fisch, } \\
\text { viele Genussmittel, Alkohol, } \\
\text { Kohlenhydrate mit hohem GI } \\
\text { (z. B. Zucker, Sirupe, Weißmehl) }\end{array}$ & $\begin{array}{l}\text { Wenig/selten, da ... } \\
\Theta \text { Schadstoffe, Stoffwechselsăuren, Oxidantien } \\
\Theta \text { Viele versteckte Kalorien, Fette und Zucker } \\
\text { sowie Tierprotein } \\
\Rightarrow \text { maximal } 1 \text { Mahlzeit pro Tag }\end{array}$ \\
\hline & 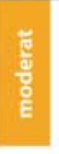 & $\begin{array}{l}\text { Komplexe Kohlenhydrate } \\
\text { mit niedrigem/mittlerem GI } \\
\text { (z. B. Vollkorngetreide), } \\
\text { Hülsenfrüchte, Nüsse, etc. }\end{array}$ & $\begin{array}{l}\text { Moderat, da ... } \\
\oplus \text { Essentielle Fettsăuren, Aminosăuren, Vitamine } \\
\oplus \text { Komplexe Kohlenhydrate, Ballaststoffe } \\
\Theta \text { Teils hohe Energiedichte } \\
\Rightarrow \text { Täglich, aber nicht zu viel }\end{array}$ \\
\hline & $\overline{\frac{g}{3}}$ & $\begin{array}{l}\text { Wasser, Kräutertee, } \\
\text { Kräuter, Gemüse, Beeren, } \\
\text { die meisten Obstsorten, etc. }\end{array}$ & $\begin{array}{l}\text { So viel wie möglich, da .... } \\
\oplus \text { Reich an Basen-, Mineralstoffen, Vitaminen, } \\
\text { Ballaststoffen, sekundären Pflanzenstoffen } \\
\oplus \text { Niedrige Energiedichte } \\
\Rightarrow \text { Grundlage jeder Mahlzeit }\end{array}$ \\
\hline
\end{tabular}

Abb. 2: Ernährungspyramide.

\section{Literatur}

[1] Arends J. Maligne Tumoren - Transketolase -like 1 (TKTL 1) - Ketogene Diät. Aktuel Ernaehr Med 2008; 33: 80-1

[2] Aune, D et al. Carbohydrates, glycemic index, glycemic load, and colorectal cancer risk: a systematic review and meta-analysis of cohort studies. Cancer Causes Control 2012 Apr; 23(4): 521-35. Epub 2012 Mar 15

[3] Bao J, Atkinson F, Petocz P, et al. Prediction of postprandial glycemia and insulinemia in lean, young, healthy adults: glycemic load compared with carbohydrate content alone. Am J Clin Nutr 2011; 93: 984-96

[4] Baron A, Migita T, Tang D, Loda M. Fatty acid synthase: a metabolic oncogene in prostate cancer? J Cell Biochem 2004; 91(1): 47-53

[5] Chajès V, Thiébaut AC, Rotival $M$, et al. Association between serum trans-monounsaturated fatty acids and breast cancer risk in the E3N-EPIC Study. Am J Epidemiol 2008; 167(11): 1312-20

[6] Danner BC, Didilis VN, Wiemeyer S, et al. Long-term survival is linked to serum LDH and partly to tumour LDH-5 in NSCLC. Anticancer Res 2010; 30(4): 1347-51

[7] Ebbesson SO, Risica PM, Ebbesson LO, Kennish JM. Eskimos have CHD despite high consumption of omega-3 fatty acids: the Alaska Siberia Project. Int I Circumpolar Health 2005; 64(4): 387-95

[8] Echtay KS, Esteves TC, Pakay JL, et al. A signalling role for 4-hydroxy-2-nonenal in regulation of mitochondrial uncoupling. EMBO 2003; 22: 4103-10

[9] Echtay KS, Murphy MP, Smith RA, et al. Superoxide activates mitochondrial uncoup- ling protein 2 from the matrix side. Studies using targeted antioxidants. J Biol Chem 2002; 277: 47129-35

[10] Elmadfa I, Leitzmann C. Ernährung des Menschen. 4. Aufl. Stuttgart: Ulmer; 2004

[11] Frattaroli J, et al. Clinical events in prostate cancer lifestyle trial: results from two years of follow-up. Urology 2008; 72(6): 1319-23

[12] Gansler TS, Hardman W, Hunt DA, et al. Increased expression of fatty acid synthase (OA-519) in ovarian neoplasms predicts shorter survival. Hum Pathol 1997; 28(6): 686-92

[13] Gonzalez CA, Riboli E. Diet and cancer prevention: Contributions from the European Prospective Investigation into Cancer and Nutrition (EPIC) study. Eur J Cancer 2010; 46 (14): 2555-62

[14] Harper ME, Antoniou A, Villalobos-Menuey $\mathrm{E}$, et al. Characterization of a novel metabolic strategy used by drug-resistant tumor cells. FASEB J 2002;16 (12): 1550-7

[15] Hunt DA, Lane HM, Zygmont ME, et al. MRNA stability and overexpression of fatty acid synthase in human breast cancer cell lines. Anticancer Res 2007; 27(1A): 27-34

[16] Ikeda T, Yoshida T, Ito Y, et al. Effect of betahydroxybutyrate and acetoacetate on insulin and glucagon secretion from perfused rat pancreas. Arch Biochem Biophys 1987; 257: 140-3

[17] Jain SK, Kannan K, Lim G. Ketosis (acetoacetate) can generate oxygen radicals and cause increased lipid peroxidation and growth inhibition in human endothelial cells. Free Radic Biol Med 1998; 25: 1083-8

[18] Jain SK, McVie R, Jaramillo JJ, Chen Y. Hyperketonemia (acetoacetate) increases the oxi- dizability of LDL+VLDL in Type-1 diabetic patients. Free Radic Biol Med 1998; 24: 175-81

[19] Jain SK, McVie R. Hyperketonemia can increase lipid peroxidation and lower glutathione levels in human erythrocytes in vitro and in Type 1 diabetic patients. Diabetes 1999; 48: 1850-5

[20] Janney NW. The metabolic relationship of the proteins to glucose. J Biol Chem 1915, 20: $321-50$

[21] Kämmerer U, Schlatterer C, Knoll G. Krebszellen lieben Zucker - Patienten brauchen Fett. Lünen: Systemed; 2011: Seite 228-35

[22] Khasawneh J, Schulz MD, Walch A, et al. Inflammation and mitochondrial fatty acid $\beta$-oxidation link obesity to early tumor promotion. Proc Natl Acad Sci USA 2009; 106 (9): 3354-9

[23] Koukourakis MI, Giatromanolaki A, Winter S, et al. Lactate dehydrogenase 5 expression in squamous cell head and neck cancer relates to prognosis following radical or postoperative radiotherapy. Oncology 2009; 77(5): 285-92

[24] Leitzmann C, Weiger M, Kurz M. Ernährung bei Krebs. München: Gräfe \& Unzer; 1996

[25] Liu Y. Fatty acid oxidation is a dominant bioenergetic pathway in prostate cancer. Prostate Cancer Prostatic Dis 2006; 9(3): 230-4

[26] Malaisse W], Lebrun P, Rasschaert J, et al. Ketone bodies and islet function: $86 \mathrm{Rb}$ handling and metabolic data. Am J Physiol 1990; 259: 123-30

[27] Malaisse W], Lebrun P, Yaylali B, et al. Ketone bodies and islet function: 45Ca handling, insulin synthesis, and release. Am J Physiol 1990; 259: 117-22 
[28] Mellanen P, Minn H, Grénman R, Härkönen P. Expression of glucose transporters in headand-neck tumors. Int J Cancer 1994; 56: 622-9

[29] Moore AE, Sabachewsky L, Toolan HW. Culture characteristics of four permanent lines of human cancer cells. Cancer Res. 1955; 15(9): 598-602

[30] Noguchi Y, Marat D, Saito A, et al. Expression of facilitative glucose transporters in gastric tumors. Hepatogastroenterology 1999; 46: 2683-9

[31] Ornish D, Magbanua MJ, Weidner G, et al. Changes in prostate gene expression in men undergoing an intensive nutrition and lifestyle intervention. Proc Natl Acad Sci USA 2008; 105(24): 8369-74

[32] Palit V, Phillips RM, Puri R, et al. Expression of HIF-1-alpha and GLUT-1 in human bladder cancer. Oncol Rep 2005; 14: 909-13

[33] Pan A, Sun Q, Bernstein AM, et al. Red meat consumption and risk of type 2 diabetes: 3 cohorts of US adults and an updated metaanalysis. Am J Clin Nutr 2011; 94(4):108896. Epub 2011 Aug 10

[34] Patel AA, Gawlinski ET, Lemieux SK, Gatenby RA. A cellular automaton model of early tumor growth and invasion. J Theor Biol. 2001; 213 (3): 315-31

[35] Pecqueur C, Bui T, Gelly C, et al. Uncoupling protein-2 controls proliferation by promoting fatty acid oxidation and limiting glycolysis-derived pyruvate utilization. FASEB J 2008; 22: 9-18

[36] Rotondo D, Davidson J. Genetics and molecular biology: fatty acid metabolism in cancer cell survival; carnitine palmitoyltransferase- 1 as a critical anticancer target. Curr Opin Lipidol 2011; 22(5): 428-9

[37] Rudlowski C, Becker A], Schroder W, et al. GLUT-1 messenger RNA and protein induction relates to the malignant transformation of cervical cancer. Am J Clin Pathol 2003; 120: 691-8

[38] Samudio I, Harmancey R, Fiegl M, et al. Pharmacologic inhibition of fatty acid oxidation sensitizes human leukemia cells to apoptosis induction. J Clin Invest 2010; 120 (1): $142-56$

[39] Santel T, Pflug G, Hemdan NY, et al. Curcumin inhibits glyoxalase 1: a possible link to its anti-inflammatory and antitumor activity. PLoS ONE 2008; 3: 3508

[40] Saxe GA, Major JM, Nguyen JY, et al. Potential attenuation of disease progression in recurrent prostate cancer with plant-based diet and stress reduction. Integr Cancer Ther 2006; 5(3): 206-13
[41] Schmidt M, Pfetzer N, Schwab M, et al. Effects of a ketogenic diet on the quality of life in 16 patients with advanced cancer: A pilot trial. Nutrition \& Metabolism 2011; 8: 54

[42] Schulz M, Hoffmann K, Weikert C, et al. Identification of a dietary pattern characterized by high-fat food choices associated with increased risk of breast cancer: the European Prospective Investigation into Cancer and Nutrition (EPIC)-Potsdam Study. Br J Nutr 2008; 100(5): 942-6

[43] Sieri S, Krogh V, Muti P, et al. Fat and protein intake and subsequent breast cancer risk in postmenopausal women. Nutr Cancer 2002; 42(1): 10-7

[44] Sluijs I, Beulens JW, van der A DL, et al. Dietary intake of total, animal, and vegetable protein and risk of type 2 diabetes in the European Prospective Investigation into Cancer and Nutrition (EPIC)-NL study. Diabetes Care 2010; 33 (1): 43-48

[45] Takeuchi M, Kimura S, Kuroda J, et al. Glyoxalase-I is a novel target against Bcr-Abl+ leukemic cells acquiring stem-like characteristics in a hypoxic environment. Cell Death Differ 2010; 17 (7): 1211-20

[46] Turner N, Bruce CR, Beale SM, et al. Excess lipid availability increases mitochondrial fatty acid oxidative capacity in muscle: evi- dence against a role for reduced fatty acid oxidation in lipid-induced insulin resistance in rodents. Diabetes 2007; 56: 2085-92

[47] Turner-McGrievy GM, Barnard ND, Scialli AR. A two-year randomized weight loss trial comparing a vegan diet to a more moderate low-fat diet. Obesity 2007 (Silver Spring); 15(9): 2276-81

[48] Walenta S, Wetterling M, Lehrke M, et al. High lactate levels predict likelihood of metastases, tumor recurrence, and restricted patient survival in human cervical cancers. Cancer Res 2000; 60: 916-21

[49] Walenta S, Mueller-Klieser WF. Lactate: mirror and motor of tumor malignancy. Semin Radiat Oncol 2004; 14 (3): 267-74

[50] Welshons WV, Grady LH, Engler KS, Judy BM. Control of proliferation of MCF-7 breast cancer cells in a commercial preparation of charcoal-stripped adult bovine serum. Breast Cancer Res Treat 1992; 23: 97-104, DOI: 10.1007/BF01831481

[51] Williams CD, Stengel J, Asike MI, et al. Prevalence of nonalcoholic fatty liver disease and nonalcoholic steatohepatitis among a largely middle-aged population utilizing ultrasound and liver biopsy: a prospective study. Gastroenterology 2011; 140(1): 124-31

\section{Summary}

Without doubt, our diet affects the initition and progression of cancer. However, opinions about the proper anti-cancer diet often go far apart. In recent years, a high-protein modification of the original ketogenic diet has been propagated as an allegedly effective and science based cancer diet. Looking at the vast majority of epidemiological and nutritional studies, this approach has to be viewed with utmost caution. The theory that a „ketogenic diet“" could „starve“ the cancer and increase survival has not been scientifically proven. It is generally known that cancer cell cultures do not grow in sugar water, but in bovine serum. The high amount of animal protein in this alleged anti cancer diet serves cancer cells as fuel and building materials, burdens the metabolism and is insulinogenic. The high fat content feeds both adipocytes and cancer cells alike and is likely to promote insulin resistance in the long run. Due to the overexpression of glucose transporters, cancer cells can still obtain glucose from the blood when the human organism has died from hypoglycemia long before. The drastic carbohydrate restriction only reduces the quality of life and increases the risk of psychological and metabolic disorders, such as they are known for extreme low carb diets. New findings show that cancer cells have an increased fat metabolism and an increased beta-oxidation and fatty acids contribute to mitochondrial uncoupling and the Warburg effect. The so called anti cancer diet is at odds with nutritional and medical gold standards.

Keywords: Cancer, ketogenic diet, glycemic index, insulin index, Warburg effect 